\title{
O Cotidiano do enfermeiro no centro de atenção psicossocial álcool e drogas III sob a perspectiva da organização do trabalho
}

The daily life of nurses at the psychosocial care center alcohol and drugs III from the perspective of work organization

La vida cotidiana de las enfermeiras em el centro de atención psicosocial alcohol y drogas III desde la perspectiva de la organización del trabajo

Christina Otaviano Pinto Larivoir ${ }^{1}$, Marcelo da Silva Alves ${ }^{1}$, Maira Buss Thofehrn², Giulia Tácilla Araújo da Silva Gondim ${ }^{1 *}$.

\section{RESUMO}

Objetivo: Compreender a organização do trabalho da equipe de enfermagem em um Centro de Atenção Psicossocial Álcool e Drogas III. Métodos: Estudo qualitativo, descritivo e exploratório desenvolvido na Zona da Mata Mineira. Os dados foram coletados de abril a novembro de $2016 \mathrm{com} 10$ profissionais da equipe de enfermagem através de entrevistas semiestruturadas. A análise dos dados foi realizada por meio da análise de conteúdo de Minayo. Resultados: Emergiram as seguintes categorias: a) organização do trabalho e ações da equipe de enfermagem no CAPS ad III e b) os descaminhos de uma prática pensada e vivida e "a profissão do jeito que tem que ser". O estudo revelou que a organização do trabalho de enfermagem no CAPS ad III, ainda reproduz o modelo biomédico que dificulta a expressão da subjetividade e da criatividade dos trabalhadores e usuários. Considerações finais: $O$ enfermeiro é um importante protagonista na transformação da organização do trabalho da enfermagem, pois exerce a gestão do cuidado, entretanto precisa incentivar novas propostas como a articulação do saber/fazer que integrem todos os profissionais.

Palavras-chave: Enfermagem, Saúde mental, Organização e administração, Serviços comunitários de saúde mental.

\begin{abstract}
Objective: To understand the work organization of the nursing staff in a Psychosocial Care Center for Alcohol and Drugs III. Methods: Qualitative, descriptive and exploratory study developed in Zona da Mata Mineira. Data were collected from April to November 2016 with 10 nursing staff professionals through semistructured interviews. Data analysis was performed using Minayo content analysis. Results: The following categories emerged: a) the work organization and actions of the nursing staff at CAPS ad III and b) the misadventures of a thought and lived practice and "the profession the way it has to be". The study revealed that the organization of nursing work in CAPS ad III still reproduces the traditional model, which hinders the expression of subjectivity and creativity of workers and users. Final considerations: The nurse is an important protagonist in the transformation of the organization of nursing work, as it exercises care management, however it needs to encourage new proposals with articulation of knowledge / do that integrate all professionals.
\end{abstract}

Keywords: Nursing, mental health, Organization e administration, Community mental health services.

1 Universidade Federal de Juiz de Fora (UFJF), Juiz de Fora - MG. *E-mail: giuliatacilla@hotmail.com

2 Universidade Federal de Pelotas (UFPel), Pelotas-RS. 


\section{RESUMEN}

Objetivo: Comprender la organización del trabajo del personal de enfermería en un Centro de Atención Psicosocial para el Alcohol y las Drogas III. Métodos: Estudio cualitativo, descriptivo y exploratorio desarrollado en Zona da Mata Mineira. Los datos se recopilaron de abril a noviembre de 2016 con 10 profesionales del personal de enfermería a través de entrevistas semiestructuradas. El análisis de datos se realizó utilizando el análisis de contenido de Minayo. Resultados: Surgieron las siguientes categorías: a) organización del trabajo y acciones del personal de enfermería en CAPS ad III b) las desventuras de una práctica pensada y vivida y "la profesión como debe ser". El estudio reveló que la organización del trabajo de enfermería en CAPS ad III todavía reproduce el modelo tradicional, lo que dificulta la expresión de la subjetividad y la creatividad de los trabajadores y usuarios. Consideraciones finales: La enfermera es un protagonista importante en la transformación de la organización del trabajo de enfermería, ya que ejerce la gestión de la atención, sin embargo, debe alentar nuevas propuestas con articulación de conocimiento / acción que integre a todos los profesionales.

Palabras clave: Enfermería, Salud mental, Organización e administración, Servicios comunitarios de salud mental.

\section{INTRODUÇÃO}

A organização é uma ferramenta da gestão/administração em todas as instituições, assim como o planejamento, coordenação e avaliação. Organizar visa ordenar a estrutura organizacional, no que tange pessoas, tecnologias e demais recursos relevantes para obter os objetivos almejados por uma instituição. Implica na divisão do trabalho, responsabilidades, hierarquia e o tipo de gestão a ser escolhida (ORO J, et al., 2019)

A institucionalização da enfermagem como profissão foi promovida por Florence Nightingale no século XIX e contribuiu com muitos conhecimentos e práticas para a administração. Florence atentou-se para a formação do enfermeiro e a educação em serviço e suas ações contribuíram para a organização da infraestrutura hospitalar.

Não focou apenas na assistência de enfermagem ao paciente, mas lançou um olhar para o ambiente, ao se preocupar com a organização dos serviços de cozinha, lavanderia, rouparia, limpeza e almoxarifado por meio da observação e supervisão. Desse modo introduziu a hierarquia, a divisão do trabalho e a disciplina na enfermagem por meio da implementação de um ensino sistematizado (SILVA TA, et al., 2019; MEDEIROS AA, et al., 2015).

Nos anos seguintes começou a ser inserido o modelo taylor-fordista no ensino e no trabalho de enfermagem. Esse modelo valorizava a descrição de técnicas, controle de materiais e ao longo do tempo o trabalho em saúde e enfermagem foi se tornando cada vez mais fragmentado e mecanicista. (PAIVA KCM e MAGESTE GS, 2018).

Essa organização do trabalho está presente em várias instituições, destacando os serviços de saúde mental. No âmbito da saúde mental, a Reforma Psiquiátrica foi um movimento que proporcionou transformações não apenas na reorganização administrativa, mas na forma de perceber a doença mental e as pessoas em sofrimento psíquico (CLEMENTINO FS, et al., 2019).

A função da instituição deixou de ser a de controlar, vigiar e disciplinar os corpos, para o desenvolvimento de estratégias e possibilidades com o sujeito em sofrimento. Desse modo, os serviços de atenção psicossocial começam a ser palco de trocas sociais, construção de relações, valorização da vida, história e das subjetividades dos indivíduos (KAPPEL VB, et al.,2020).Nessa perspectiva, surge a atenção psicossocial que preconiza o tratamento sob uma nova clínica, pautada na reinserção social, na construção da autonomia, no respeito às singularidades e protagonismo do sujeito em seu tratamento e em sua vida, tendo o Centro de Atenção Psicossocial (CAPS) como um dispositivo estratégico em saúde mental (KAPPEL VB, et al.,2020). 
As diretrizes da Portaria Gabinete do Ministro/Ministério da Saúde (GM/MS) 336/02 preconizam uma equipe de saúde para o CAPS, composta por médicos, psicólogos, assistente social, enfermeiros e técnicos de enfermagem (ALVES PF, et al., 2017).

Para atender essas transformações, a equipe de enfermagem influenciada por uma prática de cuidado norteada pelo modelo tradicional precisou adequar seu fazer cotidiano a esse serviço sendo criativa e flexível, considerando o cuidado do indivíduo, família e comunidade. Esses profissionais precisaram buscar desenvolver ações de promoção da saúde, recuperação e reinserção social, acolhimento, resgate da autonomia e da identidade do sujeito e o trabalho em equipe multiprofissional (SILVA MCM, et al., 2017).

Diante desse contexto, percebe-se que apesar da inserção dos profissionais de enfermagem no Centro de Atenção Psicossocial Álcool e Drogas III (CAPS AD III) e dos avanços proporcionados pela Reforma Psiquiátrica Brasileira, a profissão esbarra no modelo biomédico e na organização do trabalho que ainda são presentes no CAPS ad.

O estudo justifica-se pela escassez de pesquisas com essa temática e pela necessidade de refletir sobre a atual organização do trabalho de enfermagem em saúde mental e as práticas assistenciais considerando que podem dificultar a qualidade do cuidado que venha ao encontro das necessidades dos usuários. $O$ estudo objetivou compreender a organização do trabalho da equipe de enfermagem de um Centro de Atenção Psicossocial Álcool e Drogas III em uma cidade da Zona da Mata Mineira.

\section{MÉTODOS}

Trata-se de estudo descritivo e exploratório, com abordagem qualitativa. O campo de pesquisa foi um Centro de Atenção Psicossocial Álcool e Drogas III de uma cidade da Zona da Mata Mineira. Participaram da pesquisa dez profissionais de enfermagem (enfermeiros e técnicos de enfermagem) que atuavam no CAPS AD III no período matutino e vespertino. Durante a coleta de dados, havia 15 profissionais na instituição, cinco não participaram por motivos pessoais. Os critérios de inclusão foram enfermeiros e técnicos de enfermagem, que trabalham no Centro de Atenção Psicossocial Álcool e Drogas III. Os critérios de exclusão foram enfermeiros e técnicos de enfermagem que não desejaram participar da pesquisa.

O instrumento de pesquisa foi uma entrevista semiestruturada com as seguintes variáveis: caracterização dos participantes e questão norteadora: Como é a organização do trabalho da equipe de enfermagem do Centro de Atenção Psicossocial Álcool e Drogas III?

O primeiro contato da pesquisadora com os participantes ocorreu no CAPS ad III individualmente. Nesse momento foi realizado o convite, explicados os objetivos, motivos para realização da pesquisa e a possibilidade de se retirar do estudo a qualquer momento sem nenhuma consequência. Foi solicitada aos profissionais que aceitaram participar do estudo a assinatura do Termo de Consentimento Livre e Esclarecido (TCL) conforme resolução 466/2012. Portanto os dados foram coletados mediante a assinatura do Termo de Consentimento Livre e Esclarecido (TCLE), conforme resolução 466/2012, no período de agosto a novembro de 2016 por meio de entrevistas individuais gravadas em áudio (MP3), realizadas no CAPS AD III, em consultórios por ser um ambiente tranquilo e com privacidade.

As entrevistas tiveram duração de no máximo quarenta e cinco minutos, nas quais participaram apenas a pesquisadora e o participante. As informações oriundas das entrevistas foram analisadas a partir da análise de conteúdo de Minayo, por meio das etapas de ordenação dos dados em programa Microsoft Word 2007, classificação através da leitura horizontal exaustiva e posterior leitura transversal para construção das categorias analíticas. Os participantes foram tratados pela letra "E" seguidos do número correspondente à ordem das entrevistas com o intuito de preservar o anonimato e facilitar a apresentação dos recortes dos depoimentos.

Destaca-se que foram contemplados todos os requisitos éticos e legais previstos em investigações envolvendo seres humanos em consonância com as disposições regulamentadoras da Resolução 466/12 do Conselho Nacional de Saúde. A pesquisa foi aprovada pelo Comitê de Ética em Pesquisa da Universidade Federal de Juiz de Fora (UFJF) com protocolo № 1.459 .570 no dia 21 de março de 2016. 


\section{RESULTADOS E DISCUSSÃO}

\section{Caracterização Sócio demográfica}

Os dados apontam na Tabela 1 que a maioria dos profissionais era do sexo feminino, $70 \%$. Quanto à faixa etária e estado civil há predominância de profissionais de enfermagem com média de 45 anos, sendo $80 \%$ casados.

Em relação à formação, todos relataram que concluíram a graduação ou o curso técnico em enfermagem entre os anos 2004 e 2012, 90\% não possuíam especialização em saúde mental e 50\% relataram terem participado de cursos de capacitação em saúde mental sem uma abordagem específica para a enfermagem.

No que se refere à atuação profissional na saúde mental, 90\% atuam no CAPS ad há menos um ano, em razão do método de contrato com duração de um ano que confere muita rotatividade de profissionais ao serviço. Entretanto, $10 \%$ dos participantes relataram ter trabalhado em serviço de saúde mental (modelo tradicional) antes do CAPS AD e afirmaram contato com a saúde mental apenas durante a graduação ou curso técnico.

Tabela 1 - Caracterização sócio demográfica dos profissionais de enfermagem que participaram do estudo.

\begin{tabular}{|c|c|c|}
\hline Variável & $\mathbf{N}$ & Valor (\%) \\
\hline \multicolumn{3}{|l|}{ Sexo } \\
\hline Feminino & 7 & 70 \\
\hline Masculino & 3 & 30 \\
\hline \multicolumn{3}{|l|}{ Idade (anos) } \\
\hline 36 a 42 & 3 & 30 \\
\hline 43 a 48 & 5 & 50 \\
\hline 49 a 55 & 2 & 20 \\
\hline \multicolumn{3}{|l|}{ Estado Civil } \\
\hline$\overline{\text { Solteiro(a) }}$ & 2 & 20 \\
\hline Casado(a) & 8 & 80 \\
\hline \multicolumn{3}{|c|}{ Ano de Conclusão de curso } \\
\hline 2004 a 2008 & 7 & 70 \\
\hline 2009 a 2012 & 3 & 30 \\
\hline \multicolumn{3}{|c|}{ Especialização em saúde mental } \\
\hline$\overline{\operatorname{Sim}}$ & 1 & 10 \\
\hline Não & 9 & 90 \\
\hline \multicolumn{3}{|c|}{ Capacitação saúde mental } \\
\hline$\overline{\operatorname{Sim}}$ & 5 & 50 \\
\hline Não & 5 & 50 \\
\hline \multicolumn{3}{|c|}{ Experiência em saúde mental } \\
\hline$\overline{\operatorname{Sim}}$ & 1 & 10 \\
\hline Não & 9 & 90 \\
\hline
\end{tabular}

Fonte: Larivoir COP, et al., 2016.

Conforme a caracterização sócio demográfica, os profissionais entrevistados apesar de terem se formado após a implantação da Reforma Psiquiátrica ainda tiveram como campo de aprendizado hospitais com leitos psiquiátricos, sem terem conhecido um serviço substitutivo.

Esse achado reforça a necessidade de transformação na saúde mental, não apenas no modelo e na forma de perceber o sofrimento psíquico, mas na formação dos profissionais de enfermagem com o intuito de formar e capacita-los para uma prática diferenciada na perspectiva da atenção psicossocial. 


\section{Organização do trabalho e ações da equipe de enfermagem no CAPS ad III}

O CAPS ad III oferece atendimento para pessoas em sofrimento psíquico decorrente do uso de álcool e outras drogas, funciona 24 horas por dia, inclusive feriados. Incialmente é realizado uma avaliação do usuário que chega ao serviço pela equipe multiprofissional a fim de determinar o tipo de tratamento, ou seja, não intensivo, semi-intensivo, intensivo e extensão de tratamento.

O atendimento não intensivo é oferecido para usuários que não precisam de suporte contínuo da equipe e conseguem realizar suas atividades, sendo atendidos até três dias no mês. $O$ atendimento semi-intensivo é oferecido quando o sofrimento psíquico é reduziu, e houve melhora das possibilidades de relacionamento, no entanto, a pessoa ainda necessita de atenção direta da equipe para se estruturar e recuperar sua autonomia.

Já o atendimento intensivo é direcionado para usuários que necessitam de acompanhamento diário e apresentam grave sofrimento psíquico, em situação de crise ou dificuldades intensas no convívio social e familiar. A extensão de tratamento como é denominado nesse CAPS ad III, consiste no acolhimento noturno em leitos de retaguarda, no qual os usuários recebem atenção integral. Os critérios de indicação são baseados em aspectos clínicos como necessidade de desintoxicação e/ou critérios biopsicossociais como situações de crise sem intercorrência clínica grave ou comorbidades; necessidade de observação, repouso e proteção; manejo de conflitos, entre outros.

A equipe de enfermagem desse serviço é dividida em enfermeiros e técnicos de enfermagem e possuem rotinas distintas. Uma equipe com quatro plantões, isto é, dois noturnos e dois diurnos, formados por enfermeiros e técnicos de enfermagem em regime 12×36 horas. E a outra composta por enfermeiro e técnicos de enfermagem que são diaristas, de segunda-feira a sexta-feira, com jornada de trabalho de 8 horas diárias.

A equipe responsável pelo plantão diurno realiza atividades no posto de enfermagem, controle e administração de medicações, verificação de sinais vitais, registro em prontuário, assistência aos usuários que estão nos leitos de retaguarda, controlam os horários de alimentação, banho e outros. Em determinadas situações como a falta de funcionários na recepção, alguns profissionais de enfermagem foram remanejados para ajudar nesse serviço.

A equipe diarista é responsável pelas atividades cotidianas dos usuários, no qual o enfermeiro atua como técnico de referência e realiza acompanhamento individual, familiar e em grupo. Realiza acolhimento, conduz projeto terapêutico singular (PTS) junto ao usuário e família, registro em prontuário, visita domiciliar, realiza grupo educativo de saúde da mulher, roda de conversa ou espaço de palavra e matriciamento e o técnico de enfermagem o acompanha nas atividades em grupo e auxilia os demais profissionais em oficinas terapêuticas.

A equipe responsável pelo plantão noturno realiza assistência aos usuários que estão nos leitos de retaguarda no acolhimento noturno, controlam e administram medicamentos, registros em prontuário, verificam sinais vitais, controlam horários de alimentação, banho entre outros.

Frente ao exposto percebe- se a presença da divisão do trabalho de enfermagem e como cada profissional dessa equipe tem ações distintas e sem uma integração e articulação de conhecimento e informações. A divisão social do trabalho da enfermagem pode ser observada por meio das falas:

"Sou enfermeiro, mas tenho uma equipe pra liderar, supervisionar. (E10)."

"Na divisão do serviço eu fico responsável pela medicação, o outro colega que tá comigo no plantão fica pelos pacientes nos leitos (...). (E7)"

Nesse sentido, percebe-se que no CAPS a divisão do trabalho fragmenta o cuidado e distancia o líder da equipe da assistência, do estar junto. $O$ enfermeiro se responsabiliza pelo gerenciamento do serviço de enfermagem e delega ações, funções e práticas de cuidado para sua equipe, desse modo seu trabalho é exercido por técnicos de enfermagem, o que pode ser determinante para a perda da autonomia e controle do trabalho da profissão (SHOJI S, et al., 2016; MELO CMM, et al., 2016). 
A divisão do trabalho também dificulta que os profissionais de enfermagem incorporem em sua prática de cuidado tecnologias que semeiem a construção do vínculo, autonomia, trocas sociais e reinserção dos sujeitos na sociedade.

Destaca-se que as oficinas terapêuticas podem ser consideradas um tipo de tecnologia de cuidado que têm o intuito de promover qualidade de vida para as pessoas em sofrimento psíquico decorrente do uso de álcool e outras drogas, contribuir para a reinserção social, estimular a comunicação terapêutica, estimular a mente e as potencialidades do sujeito (FARIAS ID, et al., 2017; SOUZA J, et al., 2019). No entanto a equipe de enfermagem não desenvolve nenhuma oficina nesse CAPS e relatam dificuldade em fazê-lo em função do tipo de estrutura organizacional, conforme consta nos fragmentos:

"A gente tem oficina terapêutica aqui, mas não é a enfermagem que faz. É bem difícil se envolver nisso, pois a gente já tá envolvido com nosso trabalho no posto. A medicação, o banho, sinais vitais, essas coisas. (E5)"

"Esse tipo de trabalho é pra um enfermeiro só. Eu por exemplo fico responsável por gerencia os técnicos no posto. Então nem me envolvo nisso. Foi organizado dessa maneira. (E10)"

Esses espaços facilitam a interação entre usuários e profissionais e a construção do relacionamento terapêutico, no entanto em função da divisão do trabalho de enfermagem cada profissional é responsável por uma atividade e não há interação e trocas entre eles. Há uma valorização do quantitativo em relação ao qualitativo, logo que, os interesses da instituição são tratados como prioridade.

\section{Os des (caminhos) de uma prática pensada e vivida: "a profissão do jeito que tem que ser"}

Conforme apresentado na categorização sócio demográfica, a maioria dos profissionais de enfermagem teve contato com a saúde mental apenas durante a graduação e/ou curso técnico. Também relataram que no serviço de saúde mental tiveram poucas capacitações e que não foram específicas para a equipe de enfermagem. Desse modo, destaca-se a necessidade de haver capacitação específica em saúde mental para enfermagem conforme consta nos fragmentos:

"Eu estou sem rumo. Não sei a minha função. Precisa de capacitação, treinamento específico em enfermagem para a nossa equipe. (E1)"

"Poderia ter mais capacitação né? (E4)"

Observa-se que há uma incongruência com o que é preconizado pela Portaria GM no 336 e a qualificação profissional de enfermeiros no CAPS ad, uma vez que, segundo essa portaria o enfermeiro que faz parte da equipe do CAPS, deveria ter especialização em saúde mental, o que aparentemente não ocorre, conforme apresenta a categorização sócio demográfica e pode refletir na prática cotidiana (CEZAR MA e MELO CMM, 2018).

Nesse sentido percebe-se que há carência na formação de técnicos e enfermeiros em relação à saúde mental e especificamente sobre substâncias psicoativas. Os cursos ainda estão alicerçados no modelo biomédico e sua grade curricular concede pouco espaço para disciplinas como saúde mental, com carga horária reduzida e em alguns casos sem campo para prática fundamentada na rede psicossocial, o que pode influenciar na qualidade, no aprofundamento dos conhecimentos científicos e no interesse dos alunos pela disciplina, proporcionando uma formação em saúde mental deficitária que poderá refletir negativamente na prática (MOREIRA COF e DIAS MSA, 2015; TAVARES CMM, et al., 2015).

Em contrapartida, diante dos discursos pode-se perceber que os profissionais de enfermagem esperam que sejam promovidas capacitações e treinamentos em serviço no CAPS ad. No entanto a formação para saúde mental não se limita apenas às instituições de ensino e em serviço, é preciso que os profissionais tomem a iniciativa de buscar aprimoramento, seja por meio de pós-graduação, cursos de atualização e até por leitura de livros, artigos e materiais disponíveis na Internet (VARAGAS D, et al., 2018). Salienta-se que ao utilizar a Internet, o profissional deve estar ciente que deve buscar conhecimento científico em periódicos de natureza científica. 
Esse desinteresse pela busca de conhecimento específico para atuar no CAPS ad pode estar relacionado com a alta rotatividade dos profissionais nesse serviço, como elucida o depoimento de E10 e E9:

"O maior problema aqui é essa rotatividade. O contrato dura um ano só. Aí quando você está se adaptando, começando a procurar livro, curso e tal o seu contrato acaba. (E10)"

"Com essa rotatividade que tem aqui, você não consegue se envolver com o serviço né? Você entra já pensando no que vai fazer quando acabar o contrato em um ano e nem se preocupa em se especializar. (E9)"

É necessário elucidar que a cidade onde ocorreu a pesquisa realiza o processo seletivo com contratação temporária, logo que, o contrato tem duração de um ano. O trabalho temporário, "temporarywork", é parte da estratégia de flexibilização do trabalho em saúde e que nos países industrializados causa insegurança no emprego e aumento da rotatividade de trabalhadores (OLIVEIRA JSA, et al., 2018).

Desse modo, a partir dos discursos percebe-se a tendência da precarização no mercado de trabalho na instituição (SILVA RM, et al., 2020). Além de ocorrer uma quebra na construção do vínculo entre profissionais e usuários e entre a equipe multiprofissional que constantemente sofre alterações em seus membros.

Subjacente a essa discussão observa-se que há um despreparo da equipe de enfermagem que atua no CAPS ad e que ainda tem sua prática de cuidado fundamentada no modelo da biomedicina. Por falta de conhecimento e/ou pela alta rotatividade percebe-se que os profissionais ainda não incorporaram as propostas da Reforma Psiquiátrica e reproduzem atitudes e comportamentos que remetem ao modelo e organização do trabalho tradicional.

"Eu fico no posto de enfermagem com a medicação e fico lá em baixo às vezes. Se tiver oficina eu ajudo só. (E1)"

"A gente verifica a pressão, os sinais vitais. Não tem evolução, SAE, planejamento, diagnóstico de enfermagem. Anota se tiver alguma ocorrência, alguma coisa importante que seja importante anotar. E tem a medicação que é nossa responsabilidade. (E4)"

Diante do exposto, entende-se que esses profissionais estão mais propensos a desempenhar tarefas extrafuncionais dentro da dinâmica de trabalho no CAPS ad III, ocorrendo desvio das atividades preconizadas. Observa-se que a enfermagem exerce várias funções no que tange o modelo biomédico, restritos ao posto de enfermagem conforme exposto nos fragmentos e que apenas um profissional de enfermagem atua como técnico de referência. Como apresenta os depoimentos:

"Eu faço a Sistematização da enfermagem, mas não uso teoria de enfermagem. No momento que eu me vi terapeuta eu pensei: o que eu posso fazer pra eles? Nada melhor do que a consulta de enfermagem, então a SAE tá sendo importante. A gente avalia o risco de isolamento social, padrão de sono, alimentação. Orientação voltada à enfermagem, quanto à orientação prescrita, administração de medicação e cuidados de enfermagem. (E2)"

Ressalta-se que o técnico de referência é um profissional de nível superior responsável por acompanhar e conduzir o projeto terapêutico singular junto ao usuário e família que permite a construção do relacionamento terapêutico profissional-sujeito (BOMTEMPO VL e RODRIGUES CC, 2015).

Destaca-se que apenas um profissional de enfermagem assume a posição de técnico de referência, enquanto os demais permanecem institucionalizados, enraizados na lógica manicomial, responsáveis pelo posto de enfermagem, em seguir prescrições, controlar horários (alimentação, banho, medicação, sinais vitais) e executar ordens. Percebe-se que isso ocorre pela presença do modelo manicomial que influencia na forma como a organização do trabalho está estruturada. 
Nesse sentido vislumbra-se que há uma inclinação desse profissional para o modelo biopsicossocial, mas percebe-se em suas falas que em seu cotidiano, no mesmo tempo e espaço, ele realiza práticas voltadas para o modelo manicomial e psicossocial. $O$ que explicita que a saúde mental e a enfermagem vivenciam um processo de transição. Nesse contexto os profissionais de enfermagem precisam agrupar 0 seu arcabouço teórico e prática de cuidado às diretrizes do SUS e pressupostos da Reforma Psiquiátrica.

\section{CONSIDERAÇÕES FINAIS}

A organização do trabalho de enfermagem e seu processo de trabalho no âmbito da saúde mental, no CAPS ad III ainda é mecanicista, pautada na divisão do trabalho, fragmentada e hierarquizada. Esse modelo de organização dificulta a expressão da subjetividade e da criatividade dos trabalhadores e usuários. Promove insatisfação, sofrimento do profissional de enfermagem, desvalorização e prejudica a qualidade da assistência e o sucesso no tratamento. $O$ enfermeiro exerce a gestão do trabalho/cuidado e precisa incentivar a reflexão de sua equipe sobre a práxis de enfermagem, a construção de novas propostas de organização do trabalho, na qual haja articulação do saber/fazer e que todos os profissionais da equipe de enfermagem sejam inseridos e compreendidos como sujeitos participativos desse processo.

\section{REFERÊNCIAS}

1. ALVES PF, et al. Indicadores qualitativos de satisfação em saúde mental. Saúde Debate, 2017;

2. 41 (especial): $50-59$.

3. BOMTEMPO VL, RODRIGUES CC. O técnico de referencia e a saúde mental: uma reflexão a partir de uma residência multiprofissional. Revista de Educação, Ciência e Tecnologia, 2015; 2 (3):119-132.

4. CEZAR MA, MELO W. Centro de Atenção Psicossocial e território: espaço humano, comunicação e interdisciplinaridade. História, Ciências, Saúde, 2018; 25 (1): 127-142.

5. CLEMENTINO FS, et al. Atendimento integral e comunitário em saúde mental: avanços e desafios da Reforma Psiquiátrica. Trabalho, Educação e Saúde, 2019; 17(1): 1-14.

6. FARIAS ID, et al. Oficinas terapêuticas: percepção de trabalhadores dos Centros de Atenção Psicossocial. Journal of Nursing and Health, 2017; 7 (3):1-12.

7. KAPPEL VB, et al. Comunicação professional- família em um Centro de Atenção Psicossocial Infanto-juvenil: facilidades e dificuldades. Texto \& Contexto Enfermagem, 2020; 29 (1): 1-15.

8. MEDEIROS AA, et al. Teoria Ambientalista de Florence Nightingale: Uma Análise Crítica. Esc Anna Nery, $2015 ; 19$ (3): 518-24.

9. MELO CMM, et al. Autonomia profissional da enfermeira: algumas reflexões. Esc Anna Nery, $2016 ; 20$ (4): 1-6.

10. MOREIRA COF, DIAS MSA. Diretrizes Curriculares na saúde e as mudanças nos modelos de saúde e de educação. ABCS Health Science, 2015; 40 (3): 300-305.

11. OLIVEIRA JSA, et al. Trends in the job market of nurses in the view of managers. Revista Brasileira de Enfermagem, 2018; 71 (1): 148-155.

12. ORO J, et al. Do trabalho prescrito ao trabalho real da enfermagem em unidades de internação de Hospitais Universitários Federais. Texto \& Contexto Enfermagem, 2019; 28 (1):1-15.

13. PAIVA KCM, MAGESTE GS. Gestão de competências profissionais: estudo comparativo com médicos e enfermeiros de dois hospitais. RAHIS, 2018; 15(2): 49- 70.

14. SHOJI S, et al. Proposta de melhoria das condições de trabalho em uma unidade ambulatorial: perspectiva da enfermagem. Esc Anna Nery, 2016; 20 (2): 303-309.

15. SILVA MCM, et al. Redescobrindo um panorama de possibilidades: práticas de ressocialização oferecidas pelo centro de atenção psicossocial. Revista enfermagem UFPE, 2017; 11 (3): 1269-1278.

16. SILVA RM, et al. Precarização do mercado de trabalho de auxiliares e técnicos de Enfermagem no Ceará, Brasil. Ciência \& Saúde Coletiva, 2020; 25 (1): 135-145.

17. SILVA TA, et al. Identidade profissional do enfermeiro: uma revisão de literatura. Enfermeria Global, $2019 ; 1$ (54): 576- 588.

18. SOUZA J, et al. Promoção da saúde mental de mulheres: a influência da saúde física e do meio ambiente Revista Brasileira de Enfermagem, 2019; 72 (3): 191-198.

19. TAVARES CMM, et al. Percepção dos docentes sobre inovação no ensino de saúde mental. Investigação qualitativa, 2015; 1 (1): 247-250.

20. VARGAS D, et al. O ensino de enfermagem psiquiátrica e saúde mental no Brasil: Análise curricular da graduação. Texto \& Contexto Enfermagem, 2018; 27 (2): 1-9. 